\title{
Ovarian Pregnancy: A Case Report
}

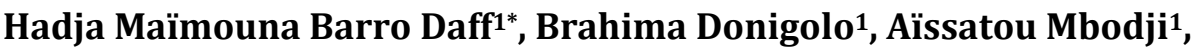 \\ Khalifa Babacar Mansour Fall'1, Youssoupha Touré1, Moussa Diallo ${ }^{1,2}$, Abdoul Aziz Diouf ${ }^{1,2}$, \\ Alassane Diouf ${ }^{1,2}$
}

${ }^{1}$ Cheikh Anta Diop University of Dakar, Gynecological and Obstetrical Clinic, Public Institution Aristide-Le Dantec Hospital, Dakar, Senegal

${ }^{2}$ Department of Obstetrics and Gynecology, University Hospital of Pikine, Thiaroye Pikine, Senegal Email: ^hmbdaff@gmail.com

How to cite this paper: Daff, H.M.B., Donigolo, B., Mbodji, A., Fall, K.B.M., Touré, Y., Diallo, M., Diouf, A.A. and Diouf, A. (2021) Ovarian Pregnancy: A Case Report. Open Journal of Obstetrics and Gynecology, 11, 1446-1451.

https://doi.org/10.4236/ojog.2021.1111134

Received: September 30, 2021

Accepted: November 7, 2021

Published: November 10, 2021

Copyright (C) 2021 by author(s) and Scientific Research Publishing Inc. This work is licensed under the Creative Commons Attribution International License (CC BY 4.0).

http://creativecommons.org/licenses/by/4.0/

\begin{abstract}
Background: Ovarian pregnancy is a rare entity of ectopic pregnancy, with a prevalence in the literature estimated between $1 / 2500$ to $1 / 5000$ births. The majority of ovarian pregnancies are diagnosed in the 1st trimester due to the noisy symptomatology. However, in small proportions, they may continue into the 2nd or 3rd trimester, making their diagnosis more difficult. Aim: We report a case of ovarian pregnancy in a 26 -year-old patient, discovered intraoperatively as a large unruptured ovarian mass. Case report: A 26-year-old woman referred to the maternity ward for management of fetal death in utero at 31 weeks of amenorrhea associated with overlying placenta previa. Ultrasound performed in the department, was in favor of an abdominal pregnancy stopped at $31 \mathrm{SA}$ with a placenta that seemed to adhere to the posterior wall of the uterus, which was empty. A Laparotomy was performed, on exploration, a large right ovarian mass was found, the site of the pregnancy. A right adnexectomy, after adhesiolysis, was performed. The incision of the operative part revealed a macerated female fetus. The clinical evolution was favorable. Conclusion: We report on a case of unruptured ovarian pregnancy, discovered in the 3rd trimester of pregnancy. The ultrasound diagnosis of an ovarian pregnancy beyond the 2 nd trimester remains a challenge, the management remains surgical.
\end{abstract}

\section{Keywords}

Ectopic Pregnancy, Ovarian Pregnancy, Adnexal Mass

\section{Introduction}

Ovarian pregnancy (OP) is an entity of ectopic pregnancy (EP), which is the development of the egg outside the uterine cavity. In the case of an ovarian preg- 
nancy, implantation takes place in the ovary. This is a rare form of ectopic pregnancy representing $3.2 \%$ of EPs, i.e., a prevalence in the literature estimated between $1 / 2500$ and $1 / 5000$ births [1]. Its primary form is rarer, occurring in $1 / 6000$ to $1 / 40,000$ pregnancies [2]. The actual physiopathology is unknown. The diagnosis of ovarian pregnancies is most often made in the first trimester on the basis of well codified anatomopathological criteria established in 1878 by Spiegelberg (91\%) [3].

The prognosis depends on the time of diagnosis and the associated signs. In fact, most of the time we can have a typical picture of ruptured ectopic pregnancy in front of a noisy symptomatology made up of pelvic pain, peritoneal irritation secondary to ovarian rupture, often with haemodynamic instability that can be life threatening. In other cases, the diagnosis is fortuitous during a laparotomy for suspicion of abdominal pregnancy with confirmation on histology with the presence of chorionic villi within the ovarian tissue.

We report here a case of unruptured ovarian pregnancy discovered in the $3 \mathrm{rd}$ trimester of pregnancy.

\section{Patient and Observation}

\subsection{Patient Information}

This was a 26-year-old primiparous patient referred to the maternity ward for management of fetal death in utero at 31 weeks of amenorrhea associated with overlying placenta previa. Her history included a cesarean section in her first pregnancy. For the current pregnancy, she had two late prenatal consultations without any particularities. A prenatal check-up was done which was without any particularities. She had benefited from a late ultrasound which found a progressive intrauterine singleton pregnancy.

\subsection{Clinical Findings and Diagnostic}

On admission, she had no complaints. The clinical examination revealed a good general condition. Her uterine height was $26 \mathrm{~cm}$. The vaginal touch was not done, because of the suspicion of placenta previa. The control ultrasound performed in the department, was in favor of an abdominal pregnancy stopped at 31 SA with a placenta that seemed to adhere to the posterior wall of the uterus, which was empty. A laparotomy was indicated and performed.

\subsection{Therapeutic Intervention and Follow-Up}

A subumbilical midline incision following the old scar was performed. On exploration, a large right ovarian mass was found, site of the pregnancy. This mass was caught in an adherent magma, notably with the omentum. The contralateral adnexa were unremarkable, as was the homolateral tube. A right adnexectomy, after adhesiolysis, was performed. The incision of the operative part revealed a macerated female fetus weighing 2023 grams (Figure 1). The postoperative course was without complications. The patient was discharged on the 3rd day of 


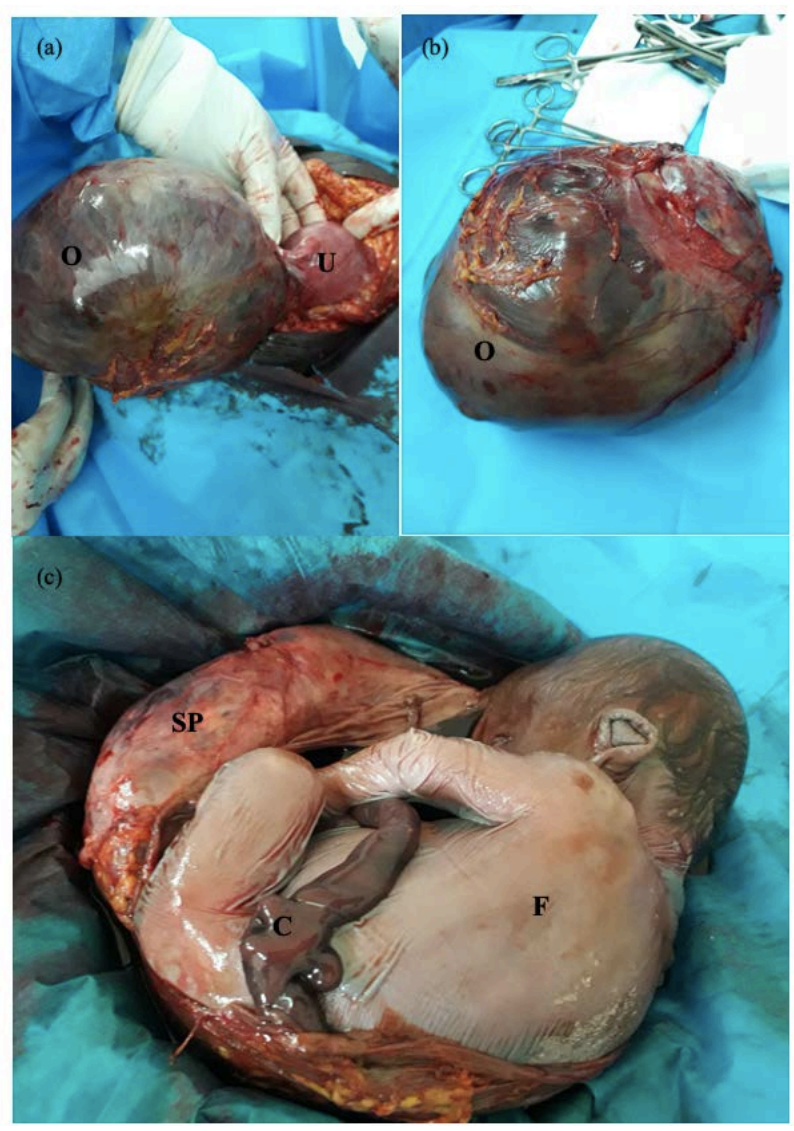

Figure 1. Intraoperative images. (a) Ovarian pregnancy connected to the uterus by the utero-ovarian ligament. (b) Ovary after adnexectomy. (c) Incision of the ovary visualizing fetus and annexes. O, ovary; U, uterus, SP, shell and placenta; F, fetus; C, umbilical cord.

her operation. Her follow-up visits, at day 15 and day 45, did not reveal any particularities. She received contraception with progestogen implants after counseling.

\subsection{Information and Consent of the Patient}

Patient informed of the interest of the case and of the publication, she gave her consent for the use of her data.

\section{Discussion}

Ovarian ectopic pregnancy results either from fertilization of an oocyte retained in the peritoneal cavity which secondarily implants on the ovarian surface, or from failure of ovulation and fertilization in the ovary [4] [5] [6] [7]. Primary ovarian pregnancy is rare, with an incidence of $1 / 6000$ to $1 / 40,000$ pregnancies [2] [6] [8]. The two main risk factors identified in the occurrence of ovarian pregnancy are contraception by intrauterine device (IUD) and in vitro fertilization (IVF) [1] [2] [6]. Indeed, an IUD is found in $57 \%$ to $90 \%$ of ovarian pregnancies. IVF is associated with $28.5 \%$ of ovarian pregnancies, thus supporting the idea of transtubal reflux of the fertilized oocyte into the ovary [2] [9]. 
Other risk factors, shared with other forms of EP, are a history of pelvic inflammatory disease, pelvic surgery, endometriosis and EP.

The only risk factor found in our patient was a cesarean section performed during her first pregnancy.

The diagnosis of an ovarian pregnancy remains a challenge but is above all clinical. Its symptomatology being almost identical to all forms of EP, we find a notion of amenorrhea, more or less associated with pelvic pain and metrorrhagia. Obstetrical ultrasound, regardless of the time of its realization, should, in addition to confirming the diagnosis of pregnancy, specify its intra or extra-uterine location. In its early form, it is often confused with a hemorrhagic corpus luteum. In its late form, 2nd or 3rd trimester, the development of the fetus can render the diagnosis of an abdominal pregnancy more difficult.

Ninety-one percent of OP are diagnosed and managed in the first trimester due to frequent ovarian rupture, $5.4 \%$ continue into the 2 nd trimester and $3.7 \%$ will reach the 3 rd trimester [3]. However, rare cases of full-term ovarian pregnancies have been reported with live child [10]. Our patient, received in the 3rd trimester, was asymptomatic, the abdominal ultrasound associated with the transvaginal ultrasound had eliminated an intrauterine pregnancy and the diagnosis retained was that of an abdominal pregnancy without specifying the exact location.

The management of ovarian pregnancies is in the majority of cases surgical, because of the frequency of ovarian rupture and therefore hemodynamic instability [3] [4]. In early forms, the standard approach is laparoscopy where conservative treatment can be considered. In advanced forms, the only possible approach is laparotomy.

Our patient had undergone an exploratory laparotomy which led to the diagnosis of an ovarian pregnancy, and an adnexectomy was then performed.

It should be remembered that the diagnostic criteria for ovarian pregnancy are based on those of Spiegelberg, which suggest that: the tube on the affected side, including the auricle, must be free of any lesion; the ovarian sac must occupy the usual anatomical place of the ovary; the ovary and the gestational sac must be connected to the uterus by the utero-ovarian ligament; there must be ovarian tissue within the ovarian sac (histological confirmation).

However, this last criterion can pose a problem in advanced pregnancies where the ovarian parenchyma is compressed, laminated and distended by fetal development, making histological examination sometimes unsatisfactory [11].

Moreover, some studies suggest that the incidence of OP could be higher if criteria other than those of Spiegelberg were taken into account. Sergent in 2002, re-evaluated these criteria, considered insufficient, with the new methods of diagnosis, treatment and follow-up of OP [3] [7].

The prognosis of $\mathrm{OP}$ is favorable if the diagnosis is made early, with appropriate management. In our patient, the postoperative course was simple, and she was discharged on day 3 after her operation. Her follow-up visits did not reveal 
any particularities. Fertility does not seem to be altered after an OP, several cases of pregnancies with favorable outcomes having been described [3] [6] [11].

\section{Conclusion}

$\mathrm{OP}$ is a rare form of EP, most often diagnosed in the 1st trimester because of the frequent ovarian rupture and the noisy symptomatology that follows. The interest of our study lies in the superposition of rare forms, in fact we find a primary, unruptured, asymptomatic GO diagnosed in the 3rd trimester of pregnancy. An early diagnosis and a quick and adapted management are essential for a favorable prognosis.

\section{Conflicts of Interest}

The authors declare no conflict of interest.

\section{References}

[1] Elwell, K.E., Sailors, J.L., Denson, P.K., Hoffman, B. and Wai, C.Y. (2015) Unruptured Second-Trimester Ovarian Pregnancy. Journal of Obstetrics and Gynaecology Research, 41, 1483-1486. https://doi.org/10.1111/jog.12726

[2] Meşeci, E., Güzel, Y., Zemheri, E., Eser, S.K., Ozkanll, S. and Kumru, P. (2013) A 34-Week Ovarian Pregnancy: Case Report and Review of the Literature. Journal of the Turkish German Gynecology Association, 14, 246-249. https://doi.org/10.5152/jtgga.2013.31391

[3] Seo, M.R., Choi, J.S., Bae, J., Lee, W.M., Eom, J.M., Lee, E. and Keum, J. (2017) Preoperative Diagnostic Clues to Ovarian Pregnancy: Retrospective Chart Review of Women with Ovarian and Tubal Pregnancy. Obstetrics \& Gynecology Science, 60, 462-468. https://doi.org/10.5468/ogs.2017.60.5.462

[4] Banys, M., K.N, Henze, C., Born, S. and Spaetling, L. (2013) A Case of Ovarian Ectopic Pregnancy after In-Vitro-Fertilization. Journal of Fertilization: In Vitro, IVF-Worldwide, Reproductive Medicine, Genetics and Stem Cell Biology, 1, 102.

[5] Tabassum, M. and Atmuri, K. (2017) The Unexpected Ovarian Pregnancy at Laparoscopy: A Review of Management. Case Reports in Obstetrics and Gynecology, 2017, Article Number: 9856802. https://doi.org/10.1155/2017/9856802

[6] Thanasa, E. and Thanasas, I. (2021) Surgical Treatment of Ovarian Pregnancy. Case Reports in Obstetrics and Gynecology, 2021, Article Number: 6618751. https://doi.org/10.1155/2021/6618751

[7] Thiam, O., Faye, P.M., Sarr, I.S.S., Seye, Y., Gueye, M.L. and Touré, A.O. (2018) Ruptured Ovarian Ectopic Pregnancy: About Two Cases. Journal of Sago (Gynecology-Obstetrics and Reproductive Health), 19, 46-50.

[8] Ge, L., Sun, W., Wang, L., Cheng, L., Geng, C., Song, Q. and Zhan, X. (2019) Ultrasound Classification and Clinical Analysis of Ovarian Pregnancy: A Study of 12 Cases. Journal of Gynecology Obstetrics and Human Reproduction, 48, 731-737. https://doi.org/10.1016/j.jogoh.2019.04.003

[9] Scutiero, G., Di Gioia, P., Spada, A. and Greco, P. (2012) Primary Ovarian Pregnancy and Its Management. JSLS, 16, 492-494. https://doi.org/10.4293/108680812X13462882736385

[10] Huang, J., Jing, X., Fan, S.Q., Zhu, F.F., Ding, Y.L., Pi, P.X., et al. (2011) Unruptured 
Full Term Ovarian Pregnancy with Live Female Infant: Case Report. Archives of Gynecology and Obstetrics, 283, 31-33.

https://doi.org/10.1007/s00404-010-1755-Z

[11] Goyal, L.D., Tondon, R., Goel, P. and Sehgal, A. (2014) Ovarian Ectopic Pregnancy: A 10 Years' Experience and Review of Literature. The Iranian Journal of Reproductive Medicine, 12, 825-830. 